\title{
Understanding VSIDS Branching Heuristics in Conflict-Driven Clause-Learning SAT Solvers
}

\author{
Jia Hui Liang, Vijay Ganesh, Ed Zulkoski, \\ Atulan Zaman, and Krzysztof Czarnecki \\ University of Waterloo, Waterloo, Canada
}

\begin{abstract}
Conflict-Driven Clause-Learning (CDCL) SAT solvers crucially depend on the Variable State Independent Decaying Sum (VSIDS) branching heuristic for their performance. Although VSIDS was proposed nearly fifteen years ago, and many other branching heuristics for SAT solving have since been proposed, VSIDS remains one of the most effective branching heuristics. Despite its widespread use and repeated attempts to understand it, this additive bumping and multiplicative decay branching heuristic has remained an enigma.

In this paper, we advance our understanding of VSIDS by answering the following key questions. The first question we pose is "what is special about the class of variables that VSIDS chooses to additively bump?" In answering this question we showed that VSIDS overwhelmingly picks, bumps, and learns bridge variables, defined as the variables that connect distinct communities in the community structure of SAT instances. This is surprising since VSIDS was invented more than a decade before the link between community structure and SAT solver performance was discovered. Additionally, we show that VSIDS viewed as a ranking function correlates strongly with temporal graph centrality measures. Putting these two findings together, we conclude that VSIDS picks high-centrality bridge variables. The second question we pose is "what role does multiplicative decay play in making VSIDS so effective?" We show that the multiplicative decay behaves like an exponential moving average (EMA) that favors variables that persistently occur in conflicts (the signal) over variables that occur intermittently (the noise). The third question we pose is "whether VSIDS is temporally and spatially focused." We show that VSIDS disproportionately picks variables from a few communities unlike, say, the random branching heuristic. We put these findings together to invent a new adaptive VSIDS branching heuristic that solves more instances than one of the best-known VSIDS variants over the SAT Competition 2013 benchmarks.
\end{abstract}

\section{Introduction}

The Boolean satisfiability (SAT) problem [14 is the quintessential NP-complete problem, a class of decision problems conjectured to be computationally hard. Yet, impressively, modern sequential Conflict-Driven Clause-Learning SAT solvers [15, 9, 32, 34, 6] are able to solve large instances obtained from real-world applications [29, 3]. Although hundreds of techniques and heuristics have been proposed 
over the last five decades to solve the Boolean SAT problem [2, 3], modern SAT solvers rely crucially only on a handful of them. Of these, the two most important are Conflict-Driven Clause-Learning with backjumping (CDCL) 34] and Variable State Independent Decaying Sum (VSIDS) branching heuristic 36. Many systematic experiments have been performed to ascertain the veracity of this observation 29. Additionally, not only is VSIDS one of the most effective branching heuristics, but many other well-known high-performing branching heuristics are simply variants of VSIDS. Researchers have proposed some theoretical explanations for the impact of clause-learning on the performance of the modern SAT solvers: clause-learning allows SAT solvers to polynomially simulate general resolution propositional proof system [39, 5, 7]. However, our understanding of the role played by VSIDS heuristic has previously been limited. The motivation for the research presented in this paper is to achieve a better scientific understanding of VSIDS. We focus on two well-known variations of VSIDS, namely cVSIDS and mVSIDS, described in Section 2 .

Our Scientific Findings and Contributions. In this paper we ask the following questions regarding the behavior of VSIDS $1{ }^{1}$ First, what is special about the class of variables that VSIDS chooses to additively bump? (Answered by Contributions I and III.) Second, what role does multiplicative decay play in making VSIDS so effective? (Answered by Contribution IV.) Third, is VSIDS temporally and spatially focused? (Answered by Contribution II.)

Contribution I: Bridge Variables and VSIDS. Community structure is a property exhibited in many real-world graphs, particularly in social networks, where the graph can be partitioned into groups of vertices, called communities, such that each group is densely connected within itself but sparsely connected with other groups. Recent research has shown that the community structure quality of the SAT input correlates with faster solving time 38. We show that bridge variables connecting distinct communities in the community structure of a SAT instance 21] are high priority targets for both the branching heuristic and clause-learning, which suggests one possible explanation for this correlation.

Contribution II: Community-focused Search and VSIDS. We define two terms, spatial focus and temporal focus, to describe how a branching heuristic focuses on certain regions of the search space during solving, with respect to the underlying community structure. We refer to this form of locality as focused search, to distinguish it from local search performed by stochastic local search solvers 25. We show that mVSIDS is more focused than cVSIDS and random branching according to these metrics.

Contribution III: Exponentially-smoothed Temporal Graph Centrality and VSIDS correlate strongly. Third, we show that VSIDS rankings correlate strongly with the variable rankings induced by exponentially smoothed temporal graph centrality (TGC) measures over the temporal variable incidence graphs (TVIG) of the original and learnt clauses of an input SAT instance. This

${ }^{1}$ All code and experimental data sets are available from our website: https://github. com/JLiangWaterloo/vsids. 
correlation remains strong throughout the run of the solver. The TVIG extends the well-known variable incidence graph over Boolean formulas by incorporating the dynamically evolving aspect of the learnt clause database inside a SAT solver and uses exponential smoothing to focus on recently learnt clauses. TGC is the temporal version of the widely-used graph centrality measures, such as degree and eigenvector centrality, which are used to identify important vertices in a graph. The definitions are inspired by recent research on temporal aspects of social networks [22, 42. For example, the timed PageRank algorithm 42. is used to discover important publications that are likely to be referenced in the future. We show that VSIDS typically selects variables with high temporal degree centrality and temporal eigenvector centrality. The above-mentioned findings essentially tell us that we have a single family of mathematically-precise graph-theoretic measures, namely TGC, that succinctly characterizes both the additive bump and multiplicative decay components of VSIDS family of heuristics. Variables that have high centrality correspond to variables in "recent" learnt clauses that are "highly-constrained" and get additively bumped. Variables that are not "persistently" highly-constrained, i.e., do not occur frequently in recent learnt clauses get decayed away quickly. Putting together Contributions I and III, we conclude that VSIDS picks high-centrality bridge variables.

Contribution IV: Exponential Moving Average and Multiplicative Decay in VSIDS. Fourth, we show that the multiplicative decay in VSIDS is a form of exponential moving average, and provide a plausible explanation as to why this is crucial to the effectiveness of VSIDS.

Contribution V: A Novel Adaptive Branching Heuristic. Our findings led to a new VSIDS called adaptVSIDS that adapatively adjusts the exponential moving average (a form of adaptive moving average) depending on the quality of the learnt clauses. We show that adaptVSIDS outperforms mVSIDS, by solving $2.4 \%$ more instances over the SAT Competition 2013 benchmarks.

\section{Background}

Here we describe VSIDS and the variable incidence graph of a CNF formula.

The VSIDS Branching Heuristic and Variants. The term VSIDS refers to a family of branching heuristics widely used in modern CDCL SAT solvers that rank all variables of a Boolean formula during the run of a solver. As things stand today, VSIDS is significantly more effective than other well-known heuristics such as DLIS [33, MOM [18], Jeroslow-Wang [28, and BOHM [12. VSIDS was a major breakthrough when first introduced as part of the Chaff solver 36. The key idea is to collect statistics over learnt clauses to guide the direction of the search, where recent learnt clauses are favored. The key characteristics of VSIDS is the additive bumping and multiplicative decay behavior, described in more details below. Another positive characteristic of VSIDS is its low computational overhead. We focus on two of the more well-known variants of VSIDS, namely, the variant from Chaff [36] and the variant from MiniSAT version 2.2.0 [15]. We 
refer to these variants as cVSIDS and mVSIDS respectively. Both variants have the common characteristics listed below.

Activity Score, Initialization and VSIDS Ranking. VSIDS assigns a floating point number, called activity, to each variable in the Boolean formula. At the begining of a run of a solver, the activity scores of all variables are typically initialized to 0 . We refer to the ranking of variables according to their activity scores in the decreasing order as the VSIDS ranking. VSIDS picks the variable with the highest activity to branch on.

Additive Bump and Multiplicative Decay. When the solver learns a clause, a set of variables is chosen, and their activities are additively increased, typically by 1 . The quantum of this increase is called the (additive) bump. At regular intervals during the run of the solver, the activities of all variables are multiplied by a constant $0<\alpha<1$ called the (multiplicative) decay factor.

cVSIDS. The activities of variables occurring in the newest learnt clause are bumped up by 1 , immediately after the clause is learnt. The activities of all variables are multiplied by a constant $0<\alpha<1$. The decay occurs after every $i$ conflicts. We follow the policy used in recent solvers like MiniSAT and use $i=1$. mVSIDS. The activities of all variables resolved during conflict analysis that lead to the learnt clause (including the variables in the learnt clause) are bumped up by 1 . The activities of all variables are decayed as in cVSIDS 2 ,

Variable Incidence Graph (VIG). The VIG of a CNF formula $F$ is defined as follows: vertices of the graph are the variables in the formula. For every clause $c \in F$ we have an edge between each pair of variables in $c$. In other words, each clause corresponds to a clique between its variables. The weight of an edge is $\frac{1}{|c|-1}$ where $|c|$ is the length of the clause. VIG does not distinguish between positive and negative occurrences of variables. We combine all edges between each pair of vertices into one weighted edge by summing the weights. More precisely, the VIG of a CNF formula $F$ is a weighted graph defined as follows: set of vertices $V=\operatorname{Var}$, set of edges $E=\{x y \mid x, y \in c \in F\}$, and the weight function $w(x y)=\sum_{x, y \in c \in F} \frac{1}{|c|-1}$.

\section{Contribution I and II: Community-focused Search, Bridge Variables, and VSIDS}

In this section, we describe the experimental setup, methodology, and results to show the connection between VSIDS and community structure.

The Hypotheses. Here we state the three hypotheses that we tested in this section: 1) Bridge Experiment: VSIDS disproportionately picks, bumps, and learns the bridge variables, 2) Spatial Focus Experiment: VSIDS disproportionately picks from a smaller number of communities rather than a large fraction

\footnotetext{
${ }^{2}$ MiniSAT's actual implementation is slightly different, but has the same effect. Rather than decaying the activities of every variable, it increases the bump quantum of all future conflicts instead [8].
} 
of the communities of a SAT instance, and 3) Temporal Focus Experiment: VSIDS typically picks from recently-seen communities.

Community Structure of the Graph of SAT Instances, and Bridge Variables. The concept of decomposing graphs into natural communities [13, 43] arose in the study of complex networks such as the graph of biological systems. Informally, a network or graph is said to have community structure if the graph can be decomposed into sub-graphs where the sub-graphs have more internal edges than outgoing edges [38. We say that a graph has a "good" community structure if the percentage of intra-community edges is significantly higher than inter-community edges. We refer to these inter-community edges as bridges, and the vertices connected by such edges as bridge vertices. In the context of the community structure of the VIG of a Boolean formula, bridge vertices are called bridge variables. We refer the reader to these papers [13, 43, for a more formal introduction to community structure of graphs.

Recently there has been some interesting discoveries regarding the impact of community on CDCL SAT solver performance 38. Specifically, the authors of the paper 38 showed that the running time of CDCL solvers is strongly correlated with community structures of SAT instances. In light of these discoveries, it was but natural for us ask the question whether VSIDS somehow exploits the community structure of SAT instances. What we discovered and explain below is that VSIDS disproportionately picks, bumps, and learns the bridge variables in the community structure of SAT instances.

Temporal and Spatial Focused Search. We further define two terms, spatial focus and temporal focus, to describe how a branching heuristic gravitates towards certain regions of the search space during solving, with respect to the underlying community structure. We say a branching heuristic is spatially focused if it disproportionately picks variables from a small set of communities, when normalized for size, throughout the entire run of the solver. A branching heuristic exhibits temporal focus if it typically picks a new decision variable from a small fixed-size window of recently-seen communities.

Experimental Setup and Methodology. Experiments were performed over the 1030 instances from SAT Competition 2013 [3], after simplification using MiniSAT simplifying-solver. We use the Louvain method [10] to compute the communities of the VIG of the input SAT formulas. There are many communitydetecting algorithms to choose from and we picked Louvain because it scales well with the size of input graphs. For each instance, the Louvain method is given an hour to compute and save the communities it finds. The community information is then given to a modified MiniSAT 2.2.0 so it can track the bridge variables. Due to the high cost, we only compute the communities once at the start.

For the Bridge-Experiment, we ran the instances using a modified MiniSAT with a timeout of 5000 seconds, as per the SAT Competition 2013 rules. Before MiniSAT begins its CDCL loop, it reads in the community information stored by the Louvain method. The solver then scans through its the initial input clauses and checks which variables share at least one clause with another variable residing in a different community and marks them as bridge variables. Whenever 
our modified version of MiniSAT 1) picks a decision variable, 2) bumps a variable, and 3) learns a clause over a variable during the search, it checks whether the variable is a bridge variable. If so, the solver updates its internal counters to keep track of the number of bridge variables in the each of the 3 scenarios. At the end of the run, the solver outputs the percentage of variables that are bridge in each of these scenarios. This additional code adds little overhead and does not change the behavior of MiniSAT. We are simply instrumenting the solver to collect statistics of interest. For the Temporal-Experiment and SpatialExperiment, we additionally modified MiniSAT to record all decision variables to a file, in order to post-process the data. We allowed a 10000 second timeout for these experiments due to this additional overhead.

The Reporting of Results. In the Bridge-Experiment, for each instance, we compute the percentage of decision variables, bumped variables, learnt clause variables, and number of variables that are also bridges. Then we averaged these percentages over the three SAT 2013 Competition benchmark categories (application, combinatorial, and random) and reported these numbers.

For the Spatial-Experiment, for every community $i$, we compute a community score $c s_{i}=$ picks_from $(i) / \operatorname{order}(i)$, where picks_from $(i)$ is the number of times the solver branched on a variable from community $i$ and $\operatorname{order}(i)$ is the size of community $i$ in terms of variables. We then use the Gini coefficient [20, a statistical measure of inequality, to compute our spatial score $s s=\operatorname{gini}\left(c_{i}\right.$ for $\mathrm{i} \in$ communities $)$. A score of 1 indicates total disparity (e.g. all picks are from one community), whereas zero indicates total equality. Higher scores therefore favor our hypothesis. We report the average ss value for each benchmark category. The intuition behind this experiment and the use of the Gini coefficient here (used in measuring the inequality of wealth distribution in countries) is that it is an effective method for computing how unequally a branching heuristic favors some communities over others. Using this metric we show for example that VSIDS disproportionately favors a small set of communities (highly unequal distribution of picks) versus random branching heuristic (largely equal distribution of picks).

For the Temporal-Experiment, we define our window size $w s$ to be $10 \%$ of the total number of communities, rounded up to the nearest integer. For all instances, our window contains the set of communities from the ws most recent decisions (note that the set may have less than $w s$ elements). At every decision, we increment a counter window_hits if the current variable is from a community in the window. We assign a temporal score $t s=$ window_hits/decisions for each instance. We report the average $t s$ value for each benchmark category. The key idea behind this experiment is to test the hypothesis that VSIDS branching favors picking from recently picked-from communities, versus random which does not display such temporal locality.

Results and Interpretations of Bridge Variable Experiment. Table 1 shows that bridge variables are highly favored in MiniSAT by its branching heuristic, conflict analysis, and clause-learning. It is a surprising result that bridge variables are favored even though the heuristics and techniques in Min- 


\begin{tabular}{|lcccc|}
\hline Category & $\begin{array}{c}\% \text { of } \\
\text { variables } \\
\text { that are bridge }\end{array}$ & $\begin{array}{c}\% \text { of } \\
\text { picked variables } \\
\text { that are bridge }\end{array}$ & $\begin{array}{c}\% \text { of } \\
\text { bumped variables } \\
\text { that are bridge }\end{array}$ & $\begin{array}{c}\text { \% of } \\
\text { learnt clause variables } \\
\text { that are bridge }\end{array}$ \\
\hline Application & 61.0 & 79.9 & 71.6 & 78.4 \\
Combinatorial & 78.2 & 87.6 & 84.3 & 88.2 \\
\hline
\end{tabular}

Table 1: MiniSAT's CDCL and mVSIDS techniques prefers to pick, bump, and learn over bridge variables.

\begin{tabular}{|l|ccc|}
\hline Category & \multicolumn{3}{|c|}{ mVSIDS cVSIDS } \\
\hline Application & 0.592 & 0.560 & 0.216 \\
Combinatorial & 0.275 & 0.261 & 0.099 \\
Random & 0.029 & 0.023 & 0.006 \\
\hline
\end{tabular}

\begin{tabular}{|l|ccc|}
\hline Category & \multicolumn{3}{|c|}{ mVSIDS cVSIDS random } \\
\hline Application & 0.580 & 0.551 & 0.268 \\
Combinatorial & 0.505 & 0.473 & 0.265 \\
Random & 0.269 & 0.268 & 0.219 \\
\hline
\end{tabular}

(a) Spatial-Experiment average ss score. (b) Temporal-Experiment average $t s$ score. Table 2: (a) VSIDS heuristics are more spatially focused than random branching.

(b) VSIDS heuristics tend to pick from recently-picked communities.

iSAT have no notion of communities. While bridge variables certainly make up a large percent of variables, the percent of picked bridge variables is even higher. Table 1 includes only the instances where the Louvain implementation completed before timing out. In total, 229/300 instances in the application category and $238 / 300$ instances in the hard combinatorial category are included in the Table 1 . In the random category, every variable is a bridge, hence the results are omitted. This is expected because it is highly improbable to generate random instances where a variable is not neighboring another variable outside its community.

Recent research suggests that CDCL solvers take advantage of good community structure in SAT instances [38] leading to faster solving time. The reason for this phenomenon is not fully understood. One possibility is that good community structure lends itself to divide-and-conquer because the bridges are easier to cut (i.e., satisfy). More precisely, the solver can focus its attention on the bridges by picking the bridge variables and assigning them appropriate values. When it eventually assigns the correct values to enough bridges, the VIG is divided into multiple components, and each component can be solved with no interference from each other. Even if the VIG cannot be completely separated, it may still be beneficial to the cut bridges between communities so that these communities can be solved relatively independently.

Results and Interpretations of Temporal and Spatial Focused Search Experiments. Table 2 a depicts the average Gini coefficient for the SpatialExperiment. Both VSIDS techniques exhibit much more inequality relative to random branching for the application and combinatorial instances, indicating that VSIDS may be attempting to hone in on certain communities. The very low values for random instances indicate that none of the branching heuristics typically favor certain communities, likely due to the poor community structures exhibited by such instances. Table $2 \mathrm{~b}$ demonstrates that VSIDS techniques are much more temporally focused on average than random branching. It is commonly believed that VSIDS improves the search locality [32, 37] which in turn improves solver performance. However, this term search locality has previously 
been not rigorously defined. We precisely defined spatial focus and temporal focus, and show that VSIDS displays high search locality in terms of these definitions.

\section{Contribution III: Experimental Evidence Supporting Strong Correlation Between TGC and VSIDS}

In this section, we describe the experiments to support the hypothesis that the VSIDS variants cVSIDS and mVSIDS, viewed as ranking functions, correlate strongly with both temporal degree centrality and temporal eigenvector centrality according to Spearman's rank correlation coefficient and top-k measures. Combining the results of this section with Contribution I (namely, VSIDS picks, bumps and learns over bridge variables), we conclude that VSIDS picks highcentrality bridge variables.

Temporal Variable Incidence Graph (TVIG). To incorporate the temporal aspect of learnt clauses we introduce temporal variable incidence graph (TVIG) here, that extends the VIG by encoding temporal information into its structure. In the TVIG, every clause is labeled with a timestamp denoted $t(c)$. The $t(c)$ is equal to 0 if $c$ is a clause from the original input formula, otherwise $t(c)$ is equal to the number conflicts up to the learning of $c$. We refer to the difference between the current time $t$ and the timestamp of a clause $t(c)$ as the age of the clause: age $(c)=t-t(c)$. Fix an exponential smoothing factor $0<\alpha<1$. The TVIG is a weighted graph constructed in the same manner as the VIG except the weight of an edge is $\frac{\alpha^{a g e(e)}}{|c|-1}$. Like the VIG, multiple edges between a pair of vertices are combined into one weighted edge. More precisely, the TVIG of a clause database at time $t$ is defined in the same way as VIG except with a modified weight function that takes the ages of clauses into account: $w(x y)=\sum_{x, y \in c \in F} \frac{\alpha^{a g e(c)}}{|c|-1}$. Observe that the TVIG evolves throughout the solving process: as new learnt clauses are added, new edges are added to the graph, and all the ages increase. As an edge's age increases, its weight decreases exponentially with time assuming no new learnt clause contains its variables. In many domains, it is often the case that more recent data points are more useful than older data points.

(Temporal) Degree and Eigenvector Centrality. A graph centrality measure is a function that assigns a real number to each vertex in a graph. The number associated with each vertex denotes its relative importance in the graph [19, 16. 41]. For example, the degree centrality [16] of a vertex in a graph is defined as the degree of the vertex. The eigenvector centrality of a vertex in a graph is defined as its corresponding value in the eigenvector of the greatest eigenvalue of the graph's adjacency matrix. We similarly define the temporal versions of degree and eigenvector centrality. The key idea needed to define temporal graph centrality measures is to incorporate temporal information inside the TVIG. The temporal degree centrality (TDC) and (resp. temporal eigenvector centrality (TEC)) of a vertex at time $t$ is defined as the degree centrality (resp. eigenvector centrality) of the vertex in the TVIG at time $t$. 
Experimental Setup and Methodology. We implemented the VSIDS variants and TGC measures in MiniSAT 2.2.0 [15. All the experiments were performed using MiniSAT on all 1030 Boolean formulas obtained from all three categories (application, combinatorial, and random) of the SAT Competition 2013 [3. Before beginning any experimentation, the instances are first simplified using MiniSAT's inbuilt preprocessor with the default settings. All experiments were performed on the SHARCNET cloud [4, where cores range in specs between 2.2 to $2.7 \mathrm{GHz}$ with $4 \mathrm{~GB}$ of memory, and 4 hour timeout. We use 100 iterations of the power iteration algorithm $[23$ to compute TEC, and 1 iteration for TDC. We use MiniSAT's default decay factor of 0.95 for VSIDS. We also use 0.95 as the exponential smoothing factor for the TVIG. We take measurements on the current state of the solver after every 5000 iterations, where an iteration is defined as a decision or a conflict. Observe that we take measurements dynamically as the solver solves an instance, and not just once at the beginning. Such a dynamic comparison gives us a much better picture of the correlation between two different ranking functions or measures than a single point of comparison.

Methodology for Comparing Rankings based on Spearman's Rank Correlation Coefficient. For each set of experiments, for each SAT instance, for every measurement made, we compute the Spearman's rank correlation coefficient 40] between the VSIDS and TGC rankings. Spearman's rank correlation coefficient is a widely-used correlation coefficient in statistics for measuring the degree of relationship between a pair of rankings. The strength of Spearman's correlation is conventionally interpreted as follows: $0.00-0.19$ is very weak, 0.20 0.39 is weak, $0.40-0.59$ is moderate, $0.60-0.79$ is strong, $0.80-1.00$ is very strong. We compute the average of the Spearman's correlation over the execution of a SAT solver on each instance. We follow the standard practice of applying the Fisher transformation [17] when aggregating the correlations.

Methodology for Comparing Rankings based on Top-k. Let $v$ be the unassigned variable with the highest ranked according to some VSIDS variant. Let $i$ be the position of variable $v$ according to a specific TGC ranking, excluding assigned variables. Then the top- $k$ measure is 1 if $i \leq k$, otherwise 0 . The rationale for this metric is that SAT solvers typically only choose the topranked unassigned variable, according to the VSIDS ranking, to branch on. If the VSIDS top-ranked unassigned variable occurs very often among the top-k ranked variables according to TGC, then we infer that VSIDS picks variables that are highly ranked according to TGC. In our experiments, we used various values for $k$. Again, we compute the average of top-k measure over the execution of a SAT solver on each instance.

The Reporting of Results. For every pair of rankings, one from the VSIDS family and the other from the TGC family, we report the top-k measure and Spearman's rank correlation coefficient between the pair of rankings every 5000 iterations. On termination, we compute the average for the instance. We take all the instance averages and average them again, and report the average of the averages. The final numbers are labeled as "mean top-k" or "mean Spearman". For example, a mean top-10 of 0.912 is interpreted as "for the average instance in the 


\begin{tabular}{|l|ccc|ccc|}
\hline & \multicolumn{3}{|c|}{ cVSIDS vs TDC } & \multicolumn{3}{c|}{ mVSIDS vs TDC } \\
& Application & Combinatorial & Random & Application & Combinatorial & Random \\
\hline Mean Spearman & 0.818 & 0.946 & 0.988 & 0.629 & 0.791 & 0.864 \\
Mean Top-1 & 0.884 & 0.865 & 0.949 & 0.427 & 0.391 & 0.469 \\
Mean Top-10 & 0.912 & 0.898 & 0.981 & 0.705 & 0.735 & 0.867 \\
\hline
\end{tabular}

Table 3: Results of comparing VSIDS and TDC.

\begin{tabular}{|l|ccc|ccc|}
\hline & \multicolumn{3}{|c|}{ cVSIDS vs TEC } & \multicolumn{3}{c|}{ mVSIDS vs TEC } \\
& Application & Combinatorial & Random & Application & Combinatorial & Random \\
\hline Mean Spearman & 0.790 & 0.926 & 0.987 & 0.675 & 0.764 & 0.863 \\
Mean Top-1 & 0.470 & 0.526 & 0.794 & 0.293 & 0.304 & 0.418 \\
Mean Top-10 & 0.693 & 0.746 & 0.957 & 0.610 & 0.670 & 0.856 \\
\hline
\end{tabular}

Table 4: Results of comparing VSIDS and TEC.

experiment, $91.2 \%$ of the measured top-ranked variables according to VSIDS are among the 10 unassigned variables with the highest centrality". Likewise, a high mean Spearman implies the average instance has a strong positive correlation between VSIDS and TGC rankings.

Results and Interpretations. In Table 3 (resp. Table 4), we compare VSIDS and TDC (resp. TEC) rankings. The data shows a strong correlation between VSIDS and TDC, in particular, the 0.818 mean Spearman between cVSIDS and TDC is high. The metrics are lower with TEC, but the correlation remains strong. mVSIDS has a better mean Spearman with TEC than TDC in the application category. We have also conducted this experiment with non-temporal degree/eigenvector centrality and the resulting mean Spearman and mean top-k are significantly lower than their temporal counterparts.

It is commonly believed that VSIDS focuses on the "most constrained part of the formula" 24, and that this is responsible for its effectiveness. However, the term "most constrained part of the formula" has previously not been welldefined in a mathematically precise manner. One intuitive way to define the constrainedness of a variable is to analyze the Boolean formula, and count how many clauses a variable occurs in. The variables can then be ranked based on this measure. In fact, this measure is the basis of the branching heuristic called DLIS [33, and was once the dominant branching heuristic in SAT solvers. We show that graph centrality measures are a good way of mathematically defining this intuitive notion of syntactic "constrainedness of variables" that has been used by the designers of branching heuristics. Degree centrality of a vertex in the VIG is indeed equal to the number of clauses it belongs to, hence it is a good basis for guessing the constrained variables for the same reason. Eigenvector centrality extends this intuition by further increasing the ranks of variables close in proximity to other constrained variables in the VIG. Additionally, as the dynamic structure of the VIG evolves due to the addition of learnt clauses by the solver, the most highly constrained variables in a given instance also change over time. Hence we incorporated learnt clauses and temporal information into the TVIG to account for changes in variables' constrainedness over time.

Besides the success of branching heuristics like VSIDS and DLIS, there is additional evidence that the syntactic structure is important for making good branching decisions. For example, Iser et al. discovered that initializing the 
VSIDS activity based on information computed on the abstract syntax tree of their translator has a positive impact on solving time [27]. In a different paper [38], the authors have shown that the graph-theoretic community structure strongly influences the running time of CDCL SAT solvers. This is more evidence of how CDCL SAT solver performance is influenced by syntactic graph properties of input formulas. Finally, by combining the results of this section with Contribution I, we conclude that VSIDS picks high-centrality bridge variables.

\section{Contribution IV: Exponential Moving Average and Multiplicative Decay}

In this section, we argue that the multiplicative decay aspect of the VSIDS branching heuristic is a form of exponential moving average (EMA) [11]. It is the inclusion of multiplicative decay in VSIDS that gives it its distinctive feature of focusing its search based on recent conflicts. The original Chaff paper 36 and patent 35] rather cryptically mentioned that VSIDS acts like a "low-pass filter". They do not specify what signals are being fed to this filter, and why the highfrequency components are being filtered out and discarded.

In his paper [8], Armin Biere was perhaps the first to articulate the idea that additive bumping of variable scores can be viewed as a signal (a square wave, to be more precise) over the run of the solver. More precisely, at every time step, the signal of a variable is 1 if it is bumped, or 0 otherwise. Armin Biere formalized normalized VSIDS [8] as $s_{n}=(1-f) \times \sum_{k=1}^{n} \delta_{k} \times f^{n-k} . s_{n}$ is the normalized VSIDS activity of a variable $v$ after the $n^{t h}$ conflict. $\delta_{k}=1$ if variable $v$ was bumped in the $k^{t h}$ conflict, otherwise $\delta_{k}=0 . f$ is the decay factor.

While Huang et al. 26] referred to VSIDS as an EMA, we will show this explicitly. We not only characterize VSIDS as an EMA explicitly, but also describe why this is crucial to the effectiveness of VSIDS as a branching heuristic. In the next section we leverage this connection between EMA and VSIDS to propose an adaptive VSIDS branching heuristic inspired by an adaptive version of EMA.

EMA is a form of exponential smoothing, used in getting rid of noise (variables whose VSIDS scores are akin to high-frequency signals) in time series data (the signals due to VSIDS scores). Exponential smoothing is a class of techniques to mitigate the effect of random noise in time series data for the purpose of analysis and forecasting. Armin Biere's normalized VSIDS equation can be rewritten to the following recursive formula: $s_{n}=(1-f) \times \delta_{n}+f \times s_{n-1}$. This formula fits exactly the definition of Brown's simple exponential smoothing, also known as exponential moving average. Therefore normalized VSIDS is exactly an EMA over the $\delta$ time series. The EMA causes VSIDS to favor variables that "persistently" occur in "recent" conflicts. A rationale why this is effective could be as follows: A conflict essentially points to faulty judgment by the solver in assigning values to variables. If a set of variables are at the root of a faulty judgment and thus occurs in a conflict, then they would repeatedly occur in related faulty judgments and hence in related conflicts. Variables that occur persistently in "recent" conflicts could be a good guess for the root cause of those conflicts. 
Hence, perhaps the most effective search strategy is to focus on determining this root cause. The learnt clauses that result from such a strategy improve in quality with time, until such time that the root cause of a set of faulty judgment has been determined and enshrined as a learnt clause.

\section{Contribution V: A Faster Branching Heuristic Based On Adaptive Moving Average}

In this section, we report on our design of a better VSIDS based on the knowledge that VSIDS decay is a form of EMA. The EMA is integral to VSIDS performance as a branching heuristic, and now that the connection between EMA and VSIDS is established, all the literature on EMA and other time series data analysis are directly applicable to VSIDS.

Adaptive Moving Average. Given that VSIDS decay is a form of EMA, we studied the literature of EMA from the financial domain [31, where it is known that the fixed decay factor can be undesirable. A moving average with a large decay factor would lag behind fast moving markets whereas a small decay factor would fail to smooth out a lot of noise. Kaufman [31] noted that a fixed decay factor performs poorly when the market volatility changes. He devised adaptive moving average where the decay factor (also known as smoothing constant) is determined by the market volatility to minimise lag and noise. By fluctuating the decay factor when necessary, adaptive moving average is better than EMA at uncovering trends in the market.

Just like how markets can go up and down, a CDCL SAT solver can go up and down in "productivity" over time. For example, Audemard and Simon 6 discovered that a learnt clause with lower literals blocks distance (LBD) [6] is of higher quality. LBD of a clause is defined to be the number of decision levels that its variables span. If the solver is in a search space that produces many learnt clauses with low LBD, then we want to encourage the solver to stay within that search space. We do so by adjusting the VSIDS decay factor to be closer to 1, i.e., decay slower. On the other hand, if the solver is in a search space that produces many learnt clauses with high LBD, it is best to choose a smaller decay factor, i.e., decay faster. Based on this insight, we devised a new VSIDS heuristic called adaptVSIDS by extending mVSIDS with an adaptive moving average. adaptVSIDS maintains a floating-point number lbdema equal to the exponential moving average of the learnt clause LBDs. Ibdema is updated after every learnt clause and this number will be used to adjust the decay factor of the variables' activities. In mVSIDS, the variables' activities are decayed by multiplying with a constant decay factor, typically 0.95 , after each conflict. Whereas in adaptVSIDS, the decay factor is adjusted based on the LBD of the learnt clause. If the LBD of the learnt clause is greater than Ibdema, then use a decay factor of 0.75 , otherwise use a decay factor of 0.99. Our website has all the code.

Experimental Setup and Methodology. The experiments were performed on the application and combinatorial categories of the SAT Competition 2013. For each instance with a timeout of 5000 seconds as per competition rules, we ran 
an unmodified MiniSAT 2.2.0 and a modified MiniSAT 2.2.0 with adaptVSIDS on StarExec [1].

Results and Interpretations. Our adaptVSIDS solved 351 instances whereas mVSIDS solved 343 instances, an increase of $2.4 \%$ more solved instances.

\section{Interpretation of Results}

We began our research by posing a series of questions regarding VSIDS, and we now interpret the results obtained in light of these questions.

What is special about the class of variables that VSIDS chooses to additively bump? (Answered by Contributions I and III.) In the bridge variables experiment (Section 3), we showed that VSIDS disproportionately favored bridge variables. Even though SAT instances have large number of bridge variables on average, the frequency with which VSIDS picks, bumps, and learns bridge variables is much higher. There is no a priori reason to believe that VSIDS would behave like this. This surprising result, plus a previous result that good community structure correlates with faster solving time [38, suggests CDCL solvers exploit community structure. More precisely, they target variables linking distinct communities, possibly as a way to solve by divide-and-conquer approach.

In the VSIDS vs. TGC experiments (Section 4), we used the Spearman's rank correlation coefficient to show that the VSIDS and TGC rankings are strongly correlated. From our experiments, we can say that for all the VSIDS variants considered in this paper, additive bumping matches with the increase in centrality of the chosen variables. We also observe from our results that the variables that solvers pick for branching have very high TGC rank. The concept of centrality allows us to define in a mathematically precise the intuition many solver developers have had, i.e., that branching on "highly constrained variables" is an effective strategy. Our bridge variable experiment combined with the TGC experiment suggests that VSIDS focuses on high-centrality bridge variables.

What role does multiplicative decay play in making VSIDS so effective? (Answered by Contribution IV, that in turn led to a new adaptive VSIDS presented as Contribution V.) We show that multiplicative decay is essentially a form of exponential smoothing (Section 5). We add an explanation as to why this is important, namely, that exponential smoothing favors variables that persistently occur in conflicts and this is a better strategy for root-cause analysis. We designed a new VSIDS technique, we call adaptVSIDS, based on the above results, wherein we rapidly decay the VSIDS activity if the learnt clause LBDs are large (Section 6). We showed that this technique is better than mVSIDS on the SAT Competition 2013 benchmark.

Is VSIDS temporally and spatially focused? (Answered by Contribution II.) We show that VSIDS exhibits spatial focus and temporal focus (Section 3), forms of locality in search. While there has been speculation among solver researchers that that CDCL with VSIDS solvers perform local search, we precisely define spatial and temporal locality in terms of the community structure. 


\section{Related Work}

Marques-Silva and Sakallah are credited with inventing the CDCL technique [34. The original VSIDS heuristic was invented by the authors of Chaff 36. Armin Biere [8] described the low-pass filter behavior of VSIDS, and Huang et al. 26] stated that VSIDS is essentially an EMA. Katsirelos and Simon 30 were the first to publish a connection between eigenvector centrality and branching heuristics. In their paper [30, the authors computed eigenvector centrality (via Google PageRank) only once on the original input clauses and showed that most of the decision variables have higher than average centrality. Also, it bears stressing that their definition of centrality is not temporal. By contrast, our results correlate VSIDS ranking with temporal degree and eigenvector centrality, and show the correlation holds dynamically throughout the run of the solver. Also, we noticed that the correlation is also significantly stronger after extending centrality with temporality. Simon and Katsirelos do hypothesize that VSIDS may be picking bridge variables (they call them fringe variables). However, they do not provide experimental evidence for this. To the best of our knowledge, we are the first to establish the following results regarding VSIDS: first, VSIDS picks, bumps, and learns high-centrality bridge variables; second, VSIDS-influenced search is more spatially and temporally focused than other branching heuristics we considered; third, explain the importance of EMA (multiplicative decay) to the effectiveness of VSIDS; and fourth, invent a new adaptive VSIDS branching heuristic based on our observations.

\section{Conclusions and Future Work}

In this paper we present various empirically-verified findings on VSIDS. We show that VSIDS tends to favor the high-centrality bridge variables in the community structure of the Boolean formula. In addition, we show that VSIDS focuses on a small subset of communities in the graph of a SAT instance during search. Lastly, we explain the multiplicative decay of VSIDS with EMA and use this finding to devise a new branching heuristic we call adaptVSIDS. These results put together show that community structure, graph centrality, and exponential smoothing are important lenses through which to understand the behavior of the VSIDS family of branching heuristics and CDCL SAT solving. In the future, we plan to strengthen our results by considering a larger number of benchmarks, solvers, branching heuristics, and graph representations.

\section{Acknowledgement}

We thank Kaveh Ghasemloo for his help in refining our TGC model and for his insight on the connection between VSIDS decay and exponential moving average. 


\section{References}

[1] Starexec, http://www.starexec.org/

[2] Proceedings of Past SAT Conferences (2013), http://www.satisfiability.org

[3] SAT Competition Website (2013), http://www.satcompetition.org

[4] SHARCNET Website (2013), https://www.sharcnet.ca

[5] Atserias, A., Fichte, J.K., Thurley, M.: Clause-learning algorithms with many restarts and bounded-width resolution. In: Theory and Applications of Satisfiability Testing-SAT 2009, pp. 114-127. Springer (2009)

[6] Audemard, G., Simon, L.: Glucose: a solver that predicts learnt clauses quality. IJCAI 9, 399-404 (2009)

[7] Beame, P., Kautz, H.A., Sabharwal, A.: Towards understanding and harnessing the potential of clause learning. Journal of Artificial Intelligence Research (JAIR) $22,319-351$ (2004)

[8] Biere, A.: Adaptive restart strategies for conflict driven SAT solvers. In: Proceedings of the 11th International Conference on Theory and Applications of Satisfiability Testing. pp. 28-33. SAT'08, Springer-Verlag, Berlin, Heidelberg (2008)

[9] Biere, A.: Lingeling (2010)

[10] Blondel, V.D., Guillaume, J.L., Lambiotte, R., Lefebvre, E.: Fast unfolding of communities in large networks. Journal of Statistical Mechanics: Theory and Experiment 2008(10), P10008 (2008)

[11] Brown, R.G.: Exponential Smoothing for predicting demand. Little (1956)

[12] Buro, M., Büning, H.K.: Report on a SAT competition. Fachbereich Math.Informatik, Univ. Gesamthochschule (1992)

[13] Clauset, A., Newman, M.E., Moore, C.: Finding community structure in very large networks. Physical review E 70(6), 066111 (2004)

[14] Cook, S.A.: The complexity of theorem-proving procedures. In: Proceedings of the Third Annual ACM Symposium on Theory of Computing. pp. 151-158. STOC '71, ACM, New York, NY, USA (1971)

[15] Een, N., Sörensson, N.: MiniSat: A SAT solver with conflict-clause minimization. Sat $5(2005)$

[16] Faust, K.: Centrality in affiliation networks. Social networks 19(2), 157-191 (1997)

[17] Fisher, R.A.: Frequency distribution of the values of the correlation coefficient in samples from an indefinitely large population. Biometrika pp. 507-521 (1915)

[18] Freeman, J.W.: Improvements to Propositional Satisfiability Search Algorithms. Ph.D. thesis, Philadelphia, PA, USA (1995), uMI Order No. GAX95-32175

[19] Freeman, L.: Centrality in social networks conceptual clarification. Social Networks 1(3), 215-239 (1979)

[20] Gini, C.: Measurement of inequality of incomes. The Economic Journal pp. 124$126(1921)$

[21] Girvan, M., Newman, M.E.: Community structure in social and biological networks. Proceedings of the National Academy of Sciences 99(12), 7821-7826 (2002)

[22] Gloor, P., Krauss, J., Nann, S., Fischbach, K., Schoder, D.: Web science 2.0: Identifying trends through semantic social network analysis. In: Computational Science and Engineering, 2009. CSE '09. International Conference on. vol. 4, pp. 215-222 (Aug 2009)

[23] Golub, G.H., Van Loan, C.F.: Matrix computations, vol. 3. JHU Press (2012)

[24] Hamadi, Y., Jabbour, S., Sais, L.: ManySAT: a parallel SAT solver. JSAT 6(4), 245-262 (2009) 
[25] Hoos, H.H., Stützle, T.: Stochastic Local Search: Foundations \& Applications. Morgan Kaufmann Publishers Inc., San Francisco, CA, USA (2004)

[26] Huang, R., Chen, Y., Zhang, W.: SAS+ planning as satisfiability. J. Artif. Int. Res. 43(1), 293-328 (Jan 2012)

[27] Iser, M., Taghdiri, M., Sinz, C.: Optimizing MiniSAT variable orderings for the relational model finder Kodkod. In: Proceedings of the 15th International Conference on Theory and Applications of Satisfiability Testing. pp. 483-484. SAT'12, Springer-Verlag, Berlin, Heidelberg (2012)

[28] Jeroslow, R.G., Wang, J.: Solving propositional satisfiability problems. Annals of mathematics and Artificial Intelligence 1(1-4), 167-187 (1990)

[29] Katebi, H., Sakallah, K.A., Marques-Silva, J.P.: Empirical study of the anatomy of modern SAT solvers. In: Proceedings of the 14th International Conference on Theory and Application of Satisfiability Testing. pp. 343-356. SAT'11, SpringerVerlag, Berlin, Heidelberg (2011)

[30] Katsirelos, G., Simon, L.: Eigenvector centrality in industrial SAT instances. In: Milano, M. (ed.) Principles and Practice of Constraint Programming, pp. 348-356. Lecture Notes in Computer Science, Springer Berlin Heidelberg (2012)

[31] Kaufman, P.J.: Trading systems and methods. John Wiley \& Sons (2013)

[32] Mahajan, Y.S., Fu, Z., Malik, S.: Zchaff2004: An efficient SAT solver. In: Proceedings of the 7th International Conference on Theory and Applications of Satisfiability Testing. pp. 360-375. SAT'04, Springer-Verlag, Berlin, Heidelberg (2005)

[33] Marques-Silva, J.P.: The impact of branching heuristics in propositional satisfiability algorithms. In: Progress in Artificial Intelligence, pp. 62-74. Springer (1999)

[34] Marques-Silva, J.P., Sakallah, K.A.: Grasp: A search algorithm for propositional satisfiability. Computers, IEEE Transactions on 48(5), 506-521 (1999)

[35] Moskewicz, M.W., Madigan, C.F., Malik, S.: Method and system for efficient implementation of boolean satisfiability (Aug 26 2008), uS Patent 7,418,369

[36] Moskewicz, M.W., Madigan, C.F., Zhao, Y., Zhang, L., Malik, S.: Chaff: Engineering an efficient SAT solver. In: Proceedings of the 38th Annual Design Automation Conference. pp. 530-535. DAC '01, ACM, New York, NY, USA (2001)

[37] Nadel, A., Ryvchin, V.: Assignment stack shrinking. In: Theory and Applications of Satisfiability Testing-SAT 2010, pp. 375-381. Springer (2010)

[38] Newsham, Z., Ganesh, V., Fischmeister, S., Audemard, G., Simon, L.: Impact of community structure on SAT solver performance. In: Theory and Applications of Satisfiability Testing-SAT 2014, pp. 252-268. Springer (2014)

[39] Pipatsrisawat, K., Darwiche, A.: On the power of clause-learning SAT solvers with restarts. In: Principles and Practice of Constraint Programming-CP 2009, pp. 654-668. Springer (2009)

[40] Spearman, C.: The proof and measurement of association between two things. The American journal of psychology 15(1), 72-101 (1904)

[41] Straffin, P.D.: Linear algebra in geography: Eigenvectors of networks. Mathematics Magazine 53(5), 269-276 (1980)

[42] Yu, P.S., Li, X., Liu, B.: Adding the temporal dimension to search - a case study in publication search. In: Skowron, A., Agrawal, R., Luck, M., Yamaguchi, T., Morizet-Mahoudeaux, P., Liu, J., Zhong, N. (eds.) Web Intelligence. pp. 543-549. IEEE Computer Society (2005)

[43] Zhang, W., Pan, G., Wu, Z., Li, S.: Online community detection for large complex networks. In: Proceedings of the Twenty-Third international joint conference on Artificial Intelligence. pp. 1903-1909. AAAI Press (2013) 


\section{A Appendix}

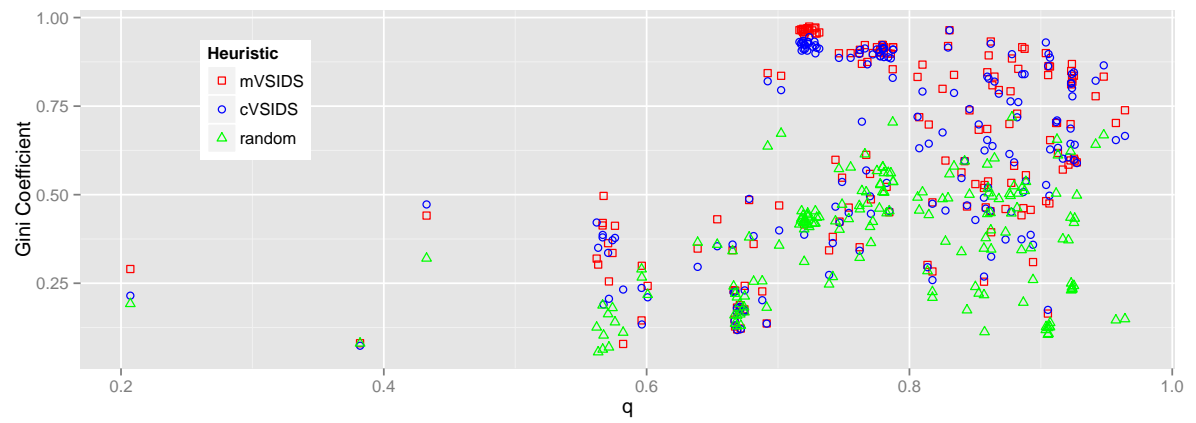

(a) Application

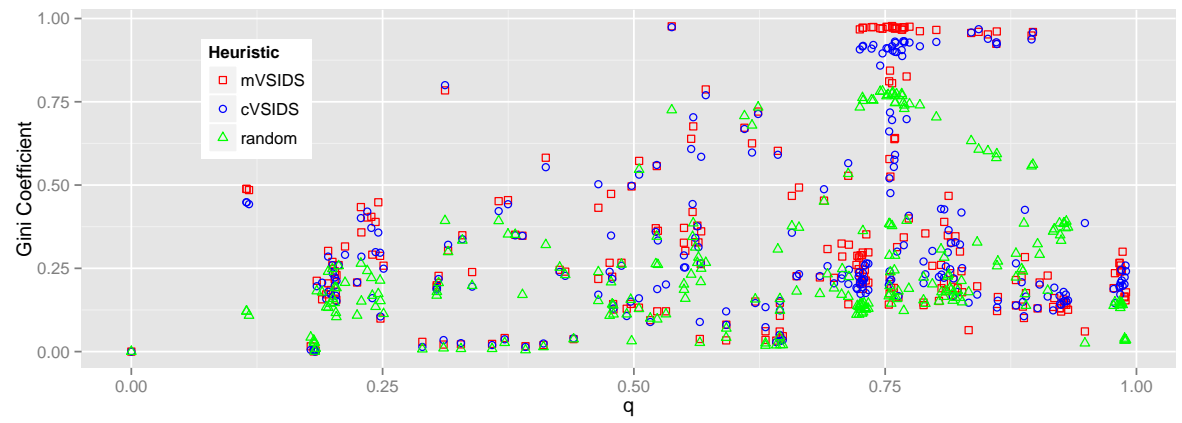

(b) Combinatorial

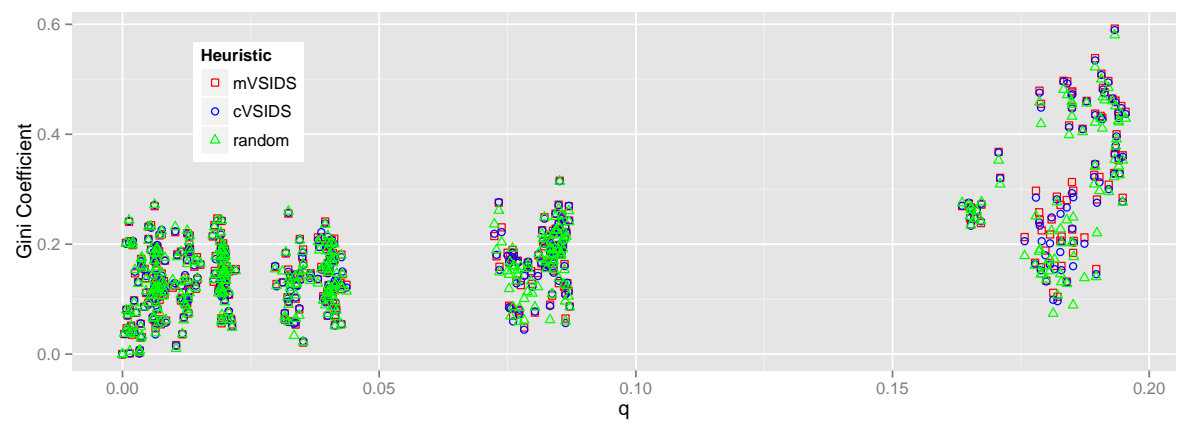

(c) Random

Fig. 1: VSIDS heuristics are much more spatially focused than random branching. For each instance and each branching heuristic, we plot the Gini coefficient of the normalized community hits (as in Section 33). Higher points indicate more spatial focus. X-axes denote the modularity of the instance's community structure - a standard metric for the quality of a community structure (higher is better). 


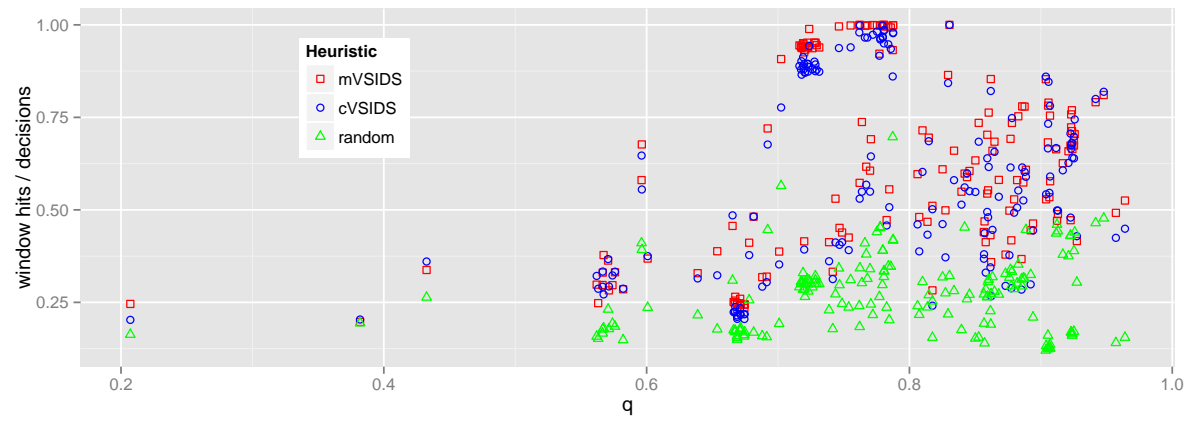

(a) Application

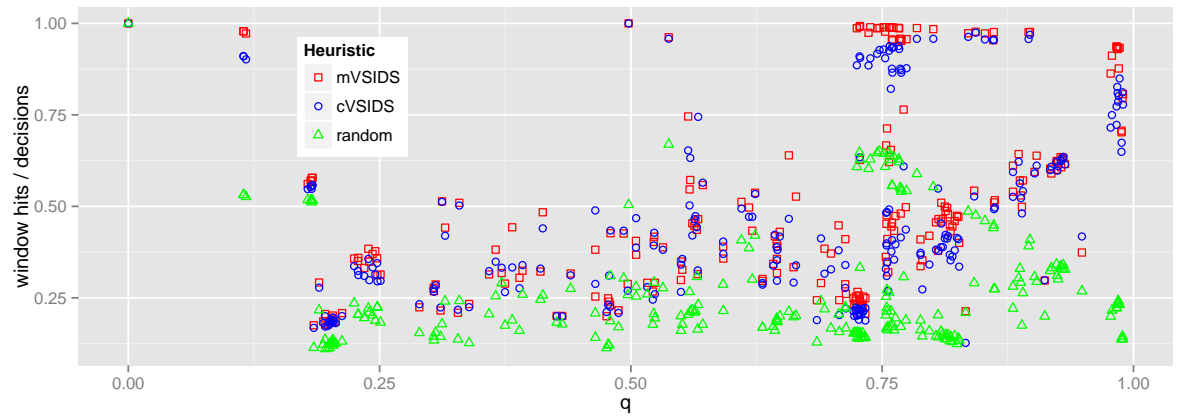

(b) Combinatorial

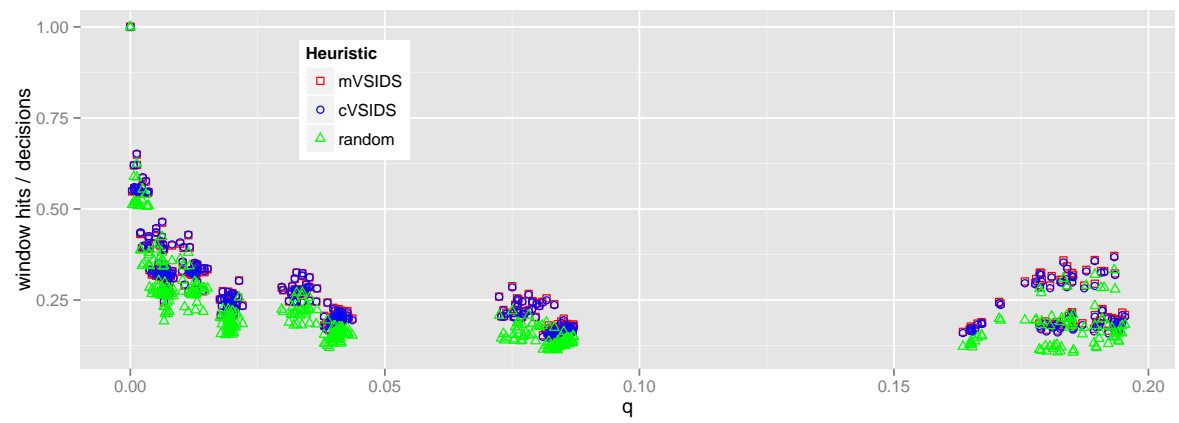

(c) Random

Fig. 2: Temporal-Experiment comparing mVSIDS, cVSIDS, and random, with a window size equal to $10 \%$ of the total number of communities (as in Section 3 ). Higher points indicate better temporal focus. Both VSIDS heuristics significantly dominate random branching, and mVSIDS is slightly more focused than cVSIDS on average. 


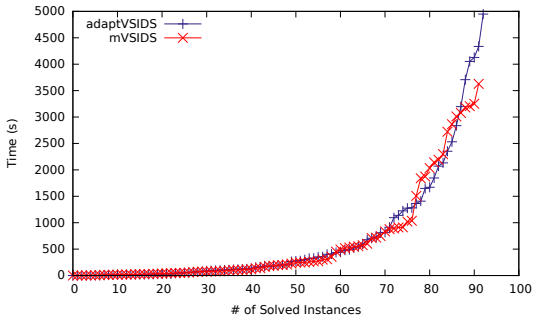

(a) satisfiable application instances

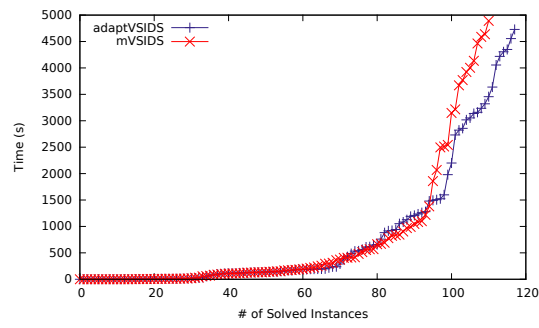

(c) satisfiable combinatorial instances (d) unsatisfiable combinatorial instances Table 5: Cactus plots for the adaptVSIDS experiment. The results are split into two categories (application or combinatorial) and two statuses (satisfiable or unsatisfiable). A point $(80,1500)$ can be interpreted as follows: there are 80 instances that take less than 1500 seconds to solve with the respective branching heuristic.

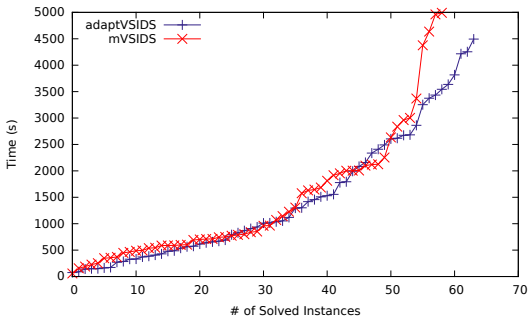

(b) unsatisfiable application instances

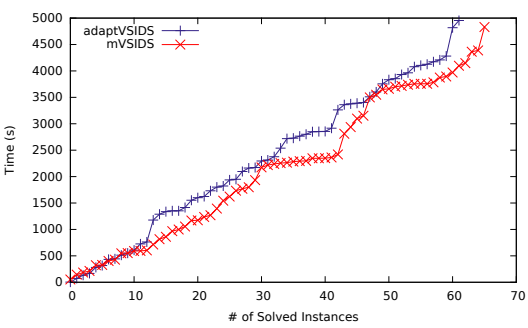

